\title{
Biocompatibility of an $x$-shaped zirconium implant in deep sclerectomy in rabbits
}

\author{
Alessia Basso • Sylvain Roy • André Mermoud
}

Received: 8 November 2007 / Revised: 22 January 2008 / Accepted: 24 January 2008 / Published online: 28 February 2008

(C) Springer-Verlag 2008

\begin{abstract}
Background The aim of this study was to evaluate the midterm biocompatibility of a new x-shaped implant made of zirconium in an animal model of glaucoma surgery.

Methods Preoperatively, ultrasound biomicroscopy (UBM), intraocular pressure (IOP) and outflow facility (OF) data were acquired. Upon surgery, one eye was chosen randomly to receive an implant, while the other received none. Ten rabbits went through a 1-, 2-, 3-, 4- and 6-month follow-up. IOP was measured regularly, UBM performed at 1,3 and 6 months after surgery. At the end of the follow-up, OF was again measured. Histology sections were analyzed.

Results For both groups IOP control was satisfactory, while $\mathrm{OF}$ initially increased at month 1 to resume preoperative values thereafter. Eyes with implants had larger filtration blebs which decreased faster than in eyes without the implant. Drainage vessel density, inflammatory cell number and fibrosis were higher in tissues near the implant.

Conclusions The zirconium implant initially promoted the positive effects of the surgery (IOP control, OF increase). Nevertheless, after several months, foreign body reactions and fibrosis had occurred on some implants that restrained the early benefit of such a procedure. Modifications of the
\end{abstract}

With the financial support of the Swiss National Science Foundation $\mathrm{N}^{\circ} 32-64103.00$

\footnotetext{
A. Basso $\cdot$ S. Roy $(\bowtie) \cdot$ A. Mermoud

Glaucoma Unit, Jules Gonin Eye Hospital,

University of Lausanne, Switzerland,

Avenue de France 15,

CH-1004 Lausanne, Switzerland

e-mail: sylvain.roy@epfl.ch
}

zirconium implant geometry could enhance the overall success rate.

Keywords Aqueous humour D Drainage device · Filtering surgery · Glaucoma · Tonometry · Ultrasonography

\section{Introduction}

Deep sclerectomy is a non-perforating filtration procedure used for the surgical treatment of open-angle glaucoma [1-7]. The key idea was to create an efficient filtration through a natural membrane without penetrating the anterior chamber. This would prevent a sudden drop of resistance, thus avoiding postoperative hypotony and other related complications $[5,6]$.

The risk of triggering a secondary fibrosis of the filtering bleb could lead to subsequent failure to efficiently control IOP. In order to maintain an effective aqueous decompression space, drainage devices can be implanted in the scleral space created during surgery [2-7]. These devices differ in material composition, size, shape, consistency, hydration ability, and whether or not they can be absorbed [2, 6, 7].

Ceramic biomaterials, like alumina and zirconium, have been used for decades in orthopaedics [8], dentistry [9, 10] and otolaryngology [11]. The biocompatibility of these materials makes them a natural choice for designing a filtering device which could prevent fibrosis of the filtering bleb and would not promote scar formation. We were interested in a neutral material onto which inflammatory cells would not adhere. The x-shape of the implant was chosen based on previous clinical results from our group with devices having the same shape [12]. The aim of this study was to evaluate the mid-term biocompatibility of a 
new $\mathrm{x}$-shaped implant made of zirconium in an animal model of glaucoma surgery.

\section{Materials \& methods}

\section{Study design}

Deep sclerectomies were performed on both eyes of ten New Zealand rabbits. The outflow facility was initially measured on each eye. One eye was randomly assigned to the implant group (deep sclerectomy with zirconium implant, DSZI), the other one to the control group (deep sclerectomy only, DS). Postoperative follow-up periods were 1, 2, 3, 4 and 6 months, and included two rabbits per period. Intraocular pressure was measured three times a week for the first 2 weeks, then once a week thereafter. Ultrasound biomicroscopy (UBM) examinations were performed preoperatively and at 1,3 and 6 months after surgery. Rabbits were killed at the end of each follow-up period. At the end of the inflation test, before the sacrifice, the outflow facility was measured a second time while ferritin dye was injected into the anterior chamber. Eyes were then enucleated and prepared for histology.

\section{Model used}

All experiments were performed on pigmented rabbits weighting 2.5 to $3.5 \mathrm{~kg}$. The animals were kept at $21^{\circ} \mathrm{C}$ in a normal 12 hours light/12 hours dark cycle. During all experiments (UBM, surgery, outflow facility measurement), general anaesthesia was performed by an intramuscular injection of a $37.5 \mathrm{mg} / \mathrm{kg}$ Ketamin and a $5.0 \mathrm{mg} / \mathrm{kg}$ Xylazine solution. At the end of the follow-up, and while still under anaesthesia, animals were sacrificed by an intravascular injection of 3-5 $\mathrm{ml}$ of a $65 \mathrm{mg} / \mathrm{ml}$ sodium pentobarbital solution. Experiments have been performed in compliance with the ARVO statement for the use of animals in ophthalmic research. (http://www.arvo.org/ AboutArvo/animalst.asp\#Recommended)

Implants

The implant was made from medical-grade zirconium (courtesy Professor Sami Sandhaus, Lausanne, Switzerland) and was designed in a $\mathrm{x}$-shape measuring $4.0 \times 4.0 \mathrm{~mm}$ by $0.8 \mathrm{~mm}$ thick (Fig. 1).

\section{Surgical procedure}

All deep sclerectomies were performed by the same experienced surgeon (SR). Details have been published previously [13]. The implant was secured onto the scleral bed with a 10/0 nylon suture. After surgery, topical application of one drop of $5 \mathrm{mg} / \mathrm{g}$ tobramycin and $1 \mathrm{mg} / \mathrm{g}$ dexamethasone (Tobradex, Alcon, Fort Worth, TX, USA) was given twice a day during the first 3 postoperative days, and once a day for the following 7 days.

\section{Intraocular pressure measurement}

Intraocular pressure measurements were performed with a TONO-Pen-XL tonometer (Mentor, Norwell, MA, USA) under a drop of local oxybuprocaïn anaesthetic (Novesin, Novartis Ophtalmics AG, Switzerland) [14]. The mean of three consecutive readings was recorded preoperatively, and three times a week postoperatively. To avoid daily and individual variation, all measurements were performed between 11 A.M. and 3 P.M. by the same investigator (AB).

\section{Ultrasound biomicroscopy}

UBM was performed to follow the evolution of the operated site [3]. The Humphrey UBM 840 system (Humphrey Instruments, Inc., San Leandro, CA, USA) used in $\mathrm{B}$ mode provided high-frequency $(50 \mathrm{MHz})$
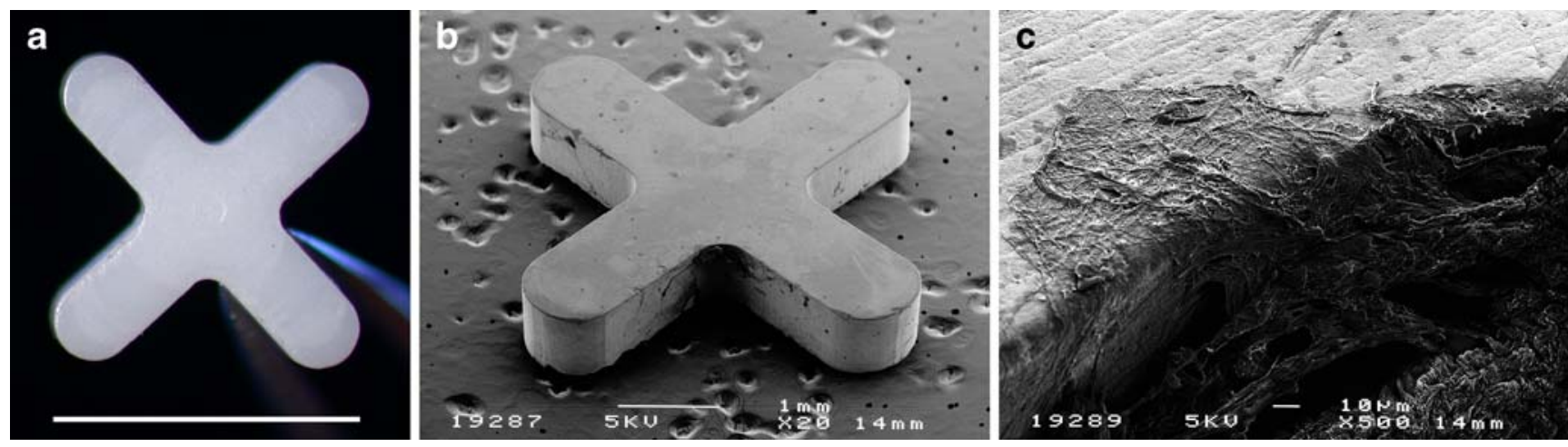

Fig. 1 a Macrophotograph of the zirconium implant. Bar: $4 \mathrm{~mm}$. b Scanning electron microscopy of the implant 6 months after surgery. c Magnification from $\mathbf{b}$ 


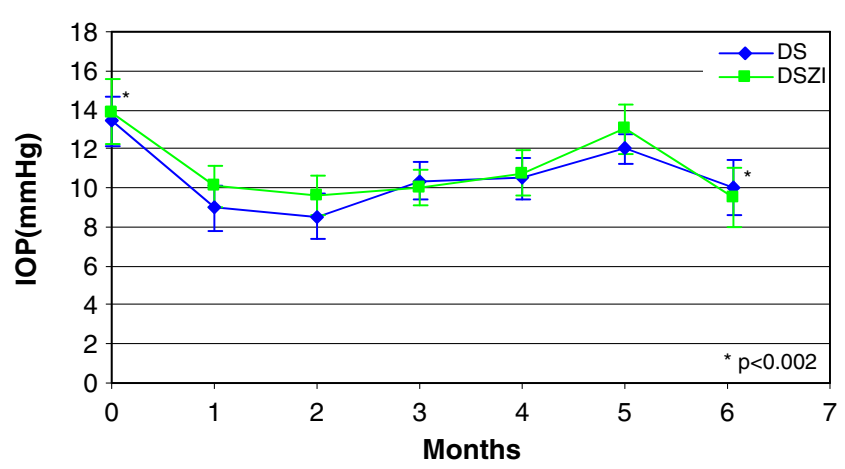

Fig. 2 Intraocular pressure profile (IOP) before and after deep sclerectomy with (DSZI) and without (DS) zirconium implant during the 6-month follow-up. The initial postoperative drop was followed by a new steady state

ultrasonic scan images. The UBM pictures were taken at the top of the filtration bleb. All examinations were performed by one single observer (AB) [15].

\section{Outflow facility measurement}

To determine the efficacy of deep sclerectomy, outflow facility was measured just before surgery and at the end of the follow-up, using an anterior chamber infusion device under constant pressure. Details have been reported previously. [13, 16]

The pressure was increased by successive $8-\mathrm{mmHg}$ steps from baseline IOP up to $32 \mathrm{mmHg}$. At each pressure level, a constant pressure was maintained using an appropriate infusion rate. Four pressure levels were recorded. Infusion flows were then plotted against pressure and a regression line was computed, the slope of the curve representing the outflow facility.

Before killing the animal, a $50 \mathrm{mg} / \mathrm{ml}$ cationic ferritin dye (horse spleen ferritin, $\mathrm{MW}=800000$, Biochemica, Fluka Chemie, Buchs, Switzerland) was injected through the catheter into the anterior chamber. While maintaining the IOP slightly over $25 \mathrm{mmHg}$, the ferritin dye was allowed to diffuse into the trabecular meshwork and the new drainage vessels for about 15 minutes.

\section{Histology}

The implants were explanted after enucleation, and classically prepared for scanning electronic microscopy. The eyes were prepared for optical microscopy, and sections were stained with haematoxylin-eosin (HE) to identify inflammatory cells. Haematoxylin-eosin plus Prussian blue revealed the ferritin in the drainage vessels, while the Goldner trichrome allowed visualization of fibrosis. [16]

Under a $10 \times$ magnification, the number of inflammatory cells and drainage vessels were counted for each eye in five consecutive sections at the surgical site and on native sclera. Results were expressed as the difference, and the ratio of vessels counted in the operated and native region. The scarring response was assessed based on the inflammatory cell density and fibrosis thickness in relation to the implant.

\section{Statistics}

The results were expressed as the mean and standard deviation (mean $\pm \mathrm{SD}$ ). Parametric comparison between means was performed using the unpaired two-tailed Student's $t$-test, and non-parametric comparison was computed with the Mann-Whitney $U$ test. Results were considered significant when $p<0.05$.

\section{Results}

Intraocular pressure

The initial post-operative pressure drop was followed by a new steady state in both groups (Fig. 2). In the zirconium
Fig. 3 Ultrasonic biomicroscopy preoperatively (a), and at 1 month post deep sclerectomy with (b) a zirconium implant. $a c$ : anterior chamber, $c$ : cornea, $c b$ : ciliary body, $i$ : iris, $i b$ : intrascleral filtration bleb, $s$ : sclera, $t d m$ : trabeculo-Descemet's membrane, $z i$ : zirconium implant
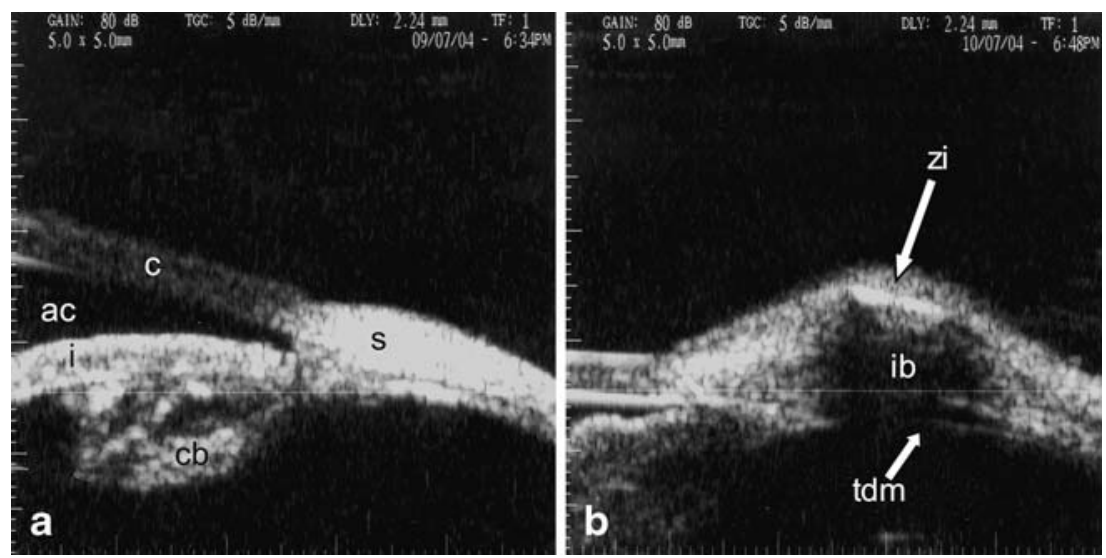
Table 1 Comparison between the mean intrascleral and subconjonctival bleb thickness $(\mathrm{mm})$ measured on UBM

\begin{tabular}{|c|c|c|c|c|c|c|}
\hline & \multicolumn{2}{|c|}{ Intrascleral bleb } & \multicolumn{2}{|c|}{ Subconjunctival bleb } & \multicolumn{2}{|l|}{$P$} \\
\hline & $\overline{\mathrm{DS}}$ & DSZI & $\overline{\mathrm{DS}}$ & DSZI & Intrascleral & Subconjunctival \\
\hline One month & $0.36( \pm 0.10)$ & $0.77( \pm 0.16)$ & $0.26( \pm 0.03)$ & $0.51( \pm 0.14)$ & $<0.001$ & 0.45 \\
\hline Three months & $0.26( \pm 0.09)$ & $0.65( \pm 0.09)$ & $0.20( \pm 0.01)$ & $0.39( \pm 0.05)$ & $<0.001$ & 0.42 \\
\hline Six months & $0.27( \pm 0.18)$ & $0.55( \pm 0.21)$ & $0.17( \pm 0.02)$ & $0.38( \pm 0.18)$ & 0.3 & 0.28 \\
\hline
\end{tabular}

Legend: deep sclerectomy with (DSZI) and without (DS) zirconium implant

implant group, IOP dropped by $25 \%$ at 6 months from a mean preoperative value of $13.4 \mathrm{mmHg}( \pm 2.46)$. In the control group, IOP dropped by $32 \%$ from a mean preoperative value of $13.9 \mathrm{mmHg}( \pm 3.35)$. The IOP drop after surgery was statistically significant in comparison with the preoperative values, $(p<0.002)$, but the difference in IOP at the end of each follow-up period between both groups was not statistically significant $(p=0.3)$.

\section{Ultrasound biomicroscopy}

The intrascleral and subconjonctival filtration blebs were visible on each surgical site for each eye of both groups at 1, 3 and 6 months postoperatively (Fig. 3). Both intrascleral and subconjonctival filtration blebs were thicker in the zirconium implant group than in the group without implant on every observation, but these differences didn't reach a significant level except for the first and third months (Table 1).

\section{Outflow facility}

A slight difference in the mean preoperative outflow facility between right $0.34 \mu \mathrm{l} / \mathrm{mmHg} \min ( \pm 0.11)$ and left $0.43 \mu \mathrm{l} / \mathrm{mmHg} \min ( \pm 0.13)$ eyes was noted, but was not considered statistically significant $(p=\mathrm{NS})$. Mean outflow facility in the control group reached maximal values during the first $(0.6 \mu \mathrm{l} / \mathrm{mmHgmin})$ and second postoperative month $(0.5 \mu \mathrm{l} / \mathrm{mmHgmin})$, and gradually decayed to reach a plateau similar to the preoperative value. On the other hand, mean outflow facility for the implant group slightly increased 1 month after surgery, and dropped to lower values than preoperative control for the rest of the study $(p=0.15)$ (Fig. 4).

\section{Histology}

In both groups, light microscopy analysis performed 1, 2, 3 and 4 months after surgery revealed the presence of a slightly higher mean number of outflow vessels in the sclera at the surgical site (10.6 for the DS group and 15.4 for the DSZI group respectively) compared to the native sclera (2.6). The difference between the surgical and native sclera was clearly significant $(p<0.001)$. After 6 months there were no longer drainage vessels visible (Table 2).
Throughout the entire follow-up, the number of drainage channels in the surgical site was higher in the DSZI group compared to the DS group, the difference being also statistically significant $(p<0.001)$. During the entire follow up, a higher number of inflammatory vessels in the surgical site compared to the native sclera were visible in both groups (Table 3) $(p<0.001)$, and this number was more important in the DSZI group than in the DS group.

The empty space of the extracted implant was lined with fibroblasts. The fibroblast reaction was already present 1 month after surgery, and increased throughout the 6-month study (Fig. 5 and Table 3). We have also noticed the presence of an inflammatory reaction, essentially composed of giant cells around the suture material, that were barely visible at 1 month, but which were more pronounced at 6 months (Fig. 5).

\section{Discussion}

Our study aims to assess the biocompatibility and efficiency of a zirconium implant in deep sclerectomy using an animal model. This implant being non-resorbable, it should be adequate to preserve an intrascleral space and to maintain good filtration, and the $\mathrm{x}$-shape should increase the volume of the filtration bleb.

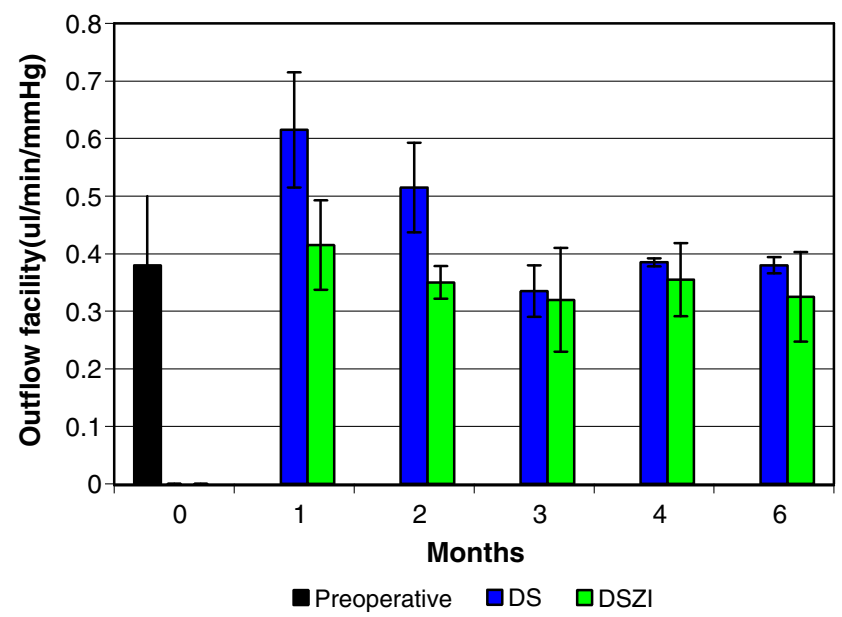

Fig. 4 Comparison of preoperative and postoperative mean outflow facility (OF) in DSZI and DS during the 6-month follow-up 
Table 2 Comparison between the mean numbers of drainage vessels observed with light microscopy. Thickness of the fibrosis around the implant. Deep sclerectomy with (DSZI) and without (DS) zirconium implant

\begin{tabular}{|c|c|c|c|c|c|c|c|}
\hline & \multicolumn{2}{|l|}{ Surgical site } & \multicolumn{2}{|c|}{ Non surgical site } & \multicolumn{2}{|c|}{ New channels } & \multirow{2}{*}{$\begin{array}{l}\text { Fibrosis }(\mu \mathrm{m}) \\
\text { DSZI }\end{array}$} \\
\hline & DS & DSZI & DS & DSZI & DS & DSZI & \\
\hline 1 month & $0.30( \pm 0.42)$ & $2.70( \pm 3.81) p<0.001$ & $0.25( \pm 0.07)$ & $0.30( \pm 0.42) \dagger$ & 0.05 & 2.4 & $131.25( \pm 8.8)$ \\
\hline 2 months & $0.25( \pm 0.35)$ & $0.80( \pm 0.28) p<0.001$ & $0.10( \pm 0.14)$ & $0.00( \pm 0.00) \dagger$ & 0.15 & 0.80 & $118.75( \pm 8.8)$ \\
\hline 3 months & $0.00( \pm 0.00)$ & $0.30( \pm 0.42) \dagger$ & $0.00( \pm 0.00)$ & $0.25( \pm 0.35) \dagger$ & 0.05 & 0.15 & $75( \pm 0.00)$ \\
\hline 4 months & $1.25( \pm 0.35)$ & $1.80( \pm 1.69) p<0.01$ & $0.50( \pm 0.48)$ & $0.00( \pm 0.00) \dagger$ & 0.75 & 1.80 & $137.5( \pm 17.68)$ \\
\hline 6 months & $0.00( \pm 0.00)$ & $0.00( \pm 0.00)$ & $0.00( \pm 0.00)$ & $0.00( \pm 0.00)$ & 0.00 & 0.00 & $162.5( \pm 53.03)$ \\
\hline
\end{tabular}

$\dot{t}=\mathrm{NS}$

Our study showed a postoperative IOP-lowering effect in both DS and DSZI groups achieved soon after surgery, followed by a slight increase 3 months after surgery. This tendency was not incredibly surprising because, firstly, the animals used were non-glaucomatous, and secondly, an adaptation to the surgically modified outflow facility was conceivable. Similarly, an increased aqueous production would counter the increased outflow facility.

While the thickness of both subconjonctival and intrascleral filtration blebs was larger in the DSZI group than the DS-only group, we still observed a faster subconjonctival and intrascleral bleb thickness reduction in the DSZI group than in the DS group, which could be explained by a inflammatory reaction that induced fibrosis.

After performing a deep sclerectomy, removing the main obstacle to aqueous humour outflow, we would have anticipated a marked and sustained increase in the outflow facility over time. This increase was noticed for both groups during the first month only. For the following 5 months, the outflow facility progressively decreased to reach preoperative values. This reduction in outflow facility could be explained by the occurrence of tissue remodelling at the surgical site or around the filtering bleb, induced or promoted by the surgery and or the implant. This reaction was apparently more important in the DSZI group.

Light microscopy showed the development of new intrascleral drainage ducts around the surgical site, which were slightly more important in the zirconium implant group, thus supporting the hypothesis that the implant was enhancing development and growth of new vessels in the surgical bed. Histological detection of drainage vessels was limited to the ferritin measurement. Ethier and Chan demonstrated in a study on human eyes that cationic ferritin reduces the outflow facility, presumably by binding to negatively charged sites in the outflow pathway, at the inner wall of Schlemm's canal [17]. To avoid methodological bias in measuring outflow facility affected by such a drawback, we have infused only for a brief period (15 minutes) in the anterior chamber, which should explain low ferritin coloration on the sections.

The drainage space was lined with spindle cells at the implant location and surrounded by fibrosis. In the deep sclerectomy-only sections, an irregular canal without spindle cells was observed. Contrary to the other non-absorbable implants (HEMA, PMMA) which are soft, the zirconium implant is very hard. This results in a huge difference in compliance when compared to the more flexible eye tissues. This strong difference between the implant and the sclera could be responsible for micro movements of the implant during the microscopic sclera expansion, and contraction during the circadian IOP changes. These movements were sufficient to induce a significant shear at the interface, resulting in a chronic inflammatory response and leading to important scarring around the zirconium implant in the later phases. The quality of the implant surface could also have played a particular role in this complication. Imperfectly

Table 3 Comparison between the mean numbers of inflammatory channels observed with light microscopy. Deep sclerectomy with (DSZI) and without (DS) zirconium implant

\begin{tabular}{|c|c|c|c|c|c|c|}
\hline & \multicolumn{2}{|l|}{ Surgical site } & \multicolumn{2}{|c|}{ Non surgical site } & \multicolumn{2}{|c|}{ New channels } \\
\hline & DS & DSZI & DS & DSZI & DS & DSZI \\
\hline 1 month & $6.05( \pm 1.48)$ & $12.90( \pm 2.69) p<0.001$ & $1.55( \pm 0.49)$ & $2.35( \pm 0.21) \mathrm{p}<0.05$ & 4.50 & 10.55 \\
\hline 2 months & $7.15( \pm 0.49)$ & $15.10( \pm 2.12) p<0.001$ & $3.25( \pm 1.06)$ & $2.30( \pm 0.14) \mathrm{p}<0.05$ & 3.95 & 12.80 \\
\hline 3 months & $5.77( \pm 0.80)$ & $13.70( \pm 0.71) p<0.001$ & $2.60( \pm 0.85)$ & $2.85( \pm 0.21) \dagger$ & 3.17 & 10.85 \\
\hline 4 months & $7.20( \pm 2.63)$ & $10.40( \pm 3.68) p<0.01$ & $1.95( \pm 0.78)$ & $1.00( \pm 0.28) \dagger$ & 5.25 & 9.40 \\
\hline 6 months & $10.60( \pm 2.26)$ & $15.37( \pm 2.30) p<0.001$ & $2.70( \pm 0.42)$ & $2.35( \pm 1.20) \dagger$ & 7.9 & 13 \\
\hline
\end{tabular}

$\digamma=\mathrm{NS}$ 


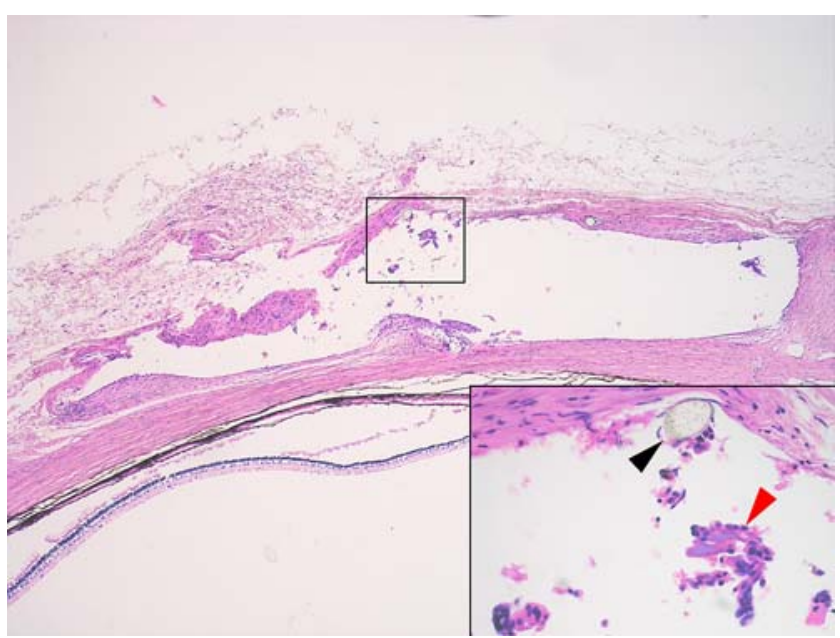

Fig. 5 Histological examination of the surgical site 6 months after DSZI showing ferritin dye (blue) in the trabeculum and drainage vessels (H\&E). Insert: giant cells (red arrow), suture material (black arrow)

polished surfaces, sharp edges or too thick an implant could have worsened the inflammatory response (Fig. 1b). The effect of the implant on the surgical site seemed to be influenced not only by the material used, which is known to have a good intrinsic biocompatibility, but also by other geometrical features causing interferences in this study. The data based on histology supports the hypothesis that the zirconium implant in our model promotes scar formation rather than preventing such reaction. In the context of the filtering surgery, such implant failed to prevent formation of fibrosis, a reported complication of the glaucoma surgery. The surface of the implant was not massively invaded by inflammatory cells (Fig. 1b), which validated the initial hypothesis that the material would not be prone to cell adhesion. Adverse events affecting the surrounding tissues could have triggered a foreign-body reaction in the presence of the implant, thus promoting the fibrosis around the device instead of limiting the extent of the scarring response.

The general conclusions of this study were limited by a few factors. These rabbits were not suffering from glaucoma, and the anatomy of their eyes was normal in all respects. The number of experiments conducted was somewhat too small to draw a definitive conclusion on glaucoma surgery with ceramic implants. Further studies should be performed to determine the real biocompatibility of this implant in glaucoma surgery.

In conclusion, this study reported results of experimental non-penetrating glaucoma surgery with zirconium implants on rabbit eyes. Initial IOP drop after surgery was followed by a slow return to preoperative values. UBM images show intrascleral and subconjunctival filtering blebs to be larger in the zirconium group. Bleb height was progressively reduced until the 6th month. Outflow facility was significantly increased in eyes having only non-penetrating surgery without implant for the first 2 months, and returned to normal thereafter. Zirconium-implanted eyes have not shown any significant increase in outflow facility after surgery. From histology, we observed that the number of drainage vessels were slightly higher for the zirconium group compared to the group without the implant. Fibrosis, scar formation and inflammatory response were significantly more pronounced in the zirconium group. These reactions were probably the main reason for the relative failure in this implant surgery despite well-known biocompatibility of this material in bioprosthesis. As it stands, this implant failed to prevent the scarring response of the filtering bleb in an experimental model of glaucoma surgery. Modifications of the zirconium implant geometry could enhance the overall success rate.

Acknowledgments We thank M. Tyler Thacher from the Lausanne Swiss Federal Institute of Technology for carefully reading and correcting the paper. The study was supported by the Swiss National Science Foundation, grant \# 32-64103.00.

\section{References}

1. Fyodorov SN, Koslov VI, Timoshkina NT et al (1990) Nonpenetrating deep sclerectomy in open angle glaucoma. Ophthalmosurg 3:52-55

2. Kozlov VI, Bagrov SN, Anisimova SY (1990) Nonpenetrating deep sclerectomy with collagen. Ophthal Surg 3:44-46

3. Chiou AG, Mermoud A, Underdahl JP, Schnyder CC (1998) An ultrasound biomicroscopic study of eyes after deep sclerectomy with collagen implant. Ophthalmology 105:746-750

4. Karlen ME, Sanchez E, Schnyder CC, Sickenberg M, Mermoud A (1999) Deep sclerectomy with collagen implant: medium term results. Br J Ophthalmol 83:6-11

5. Mermoud A, Schnyder CC, Sickenberg M, Chiou AG, Hediger SE, Faggioni R (1999) Comparison of deep sclerectomy with collagen implant and trabeculectomy in open-angle glaucoma. J Cataract Refract Surg 25:323-331

6. Shaarawy T, Nguyen C, Schnyder C, Mermoud A (2004) Comparative study between deep sclerectomy with and without collagen implant: long-term follow-up. Br J Ophthalmol 88:95-98

7. Sourdille P, Santiago PY, Ducourneau Y (1999) Non perforating surgery of the trabeculum with reticulated hyaluronic acid implant. J Fr Ophthalmol 22:794-797

8. Piconi C, Maccauro G (1999) Zirconia as a ceramic biomaterial. Biomaterials 20:1-25

9. Seghi R, Denry I, Rosentiel S (1995) Relative fracture toughness and hardness of new dental ceramics. J Prosthet Dent 74:145-150

10. Guazzato M, Albakri M, Ringer SP, Swain MV (2004) Strength, fracture toughness and microstructure of a selection of all-ceramic materials. Part I. Pressable and alumina glass-infiltrated ceramics. Dent Mater 5:441-448

11. Schadel A, Thun G, Stork L, Metzla R (1993) Immunodiffusion and immunohistochemical investigations on the reactivity of oxide ceramic middle ear implants. ORL J Otorhinolaryngol Relat Spec $55: 216-221$

12. Mansouri K, Shaarawy T, Wedrich A, Mermoud A (2006) Comparing polymethylmethacrylate implant with collagen im- 
plant in deep sclerectomy: a randomized controlled trial. J Glaucoma 15:264-270

13. Nguyen C, Boldea RC, Roy S, Schaarawy T, Uffer S, Mermoud A (2006) Outflow mechanisms after deep sclerectomy with two different designs of collagen implant in an animal model. Graefes Arch Clin Exp Ophthalmol 244:1659-1667

14. Mermoud A, Baerveld G, Minckler DS, Lee MB, Rao NA (1995) Measurement of rabbit intraocular pressure with the Tonopen. Ophthalmologica 9:275-277
15. Tello C, Liebmann J, Potash SD, Cohen H, Ritch R (1994) Measurement of biomicroscopy images: intraobserver reliability. Invest Ophthalmol Vis Sci 35:3549-3552

16. Delarive T, Mermoud A, Uffer S, Rossier A (2003) Histological findings of deep sclerectomy with collagen implant in an animal model. Br J Ophthalmol 87:1340-1344

17. Ethier RC, Chan DW (2001) Cationic ferritin changes outflow facility in human eyes whereas anionic ferritin does not. Invest Ophthalmol Vis Sci 42:1795-1802 